\title{
Synthesis of Pyrazolyl Methylene Bis Indoles by using Recyclable Nano Copper Ferrite Catalyst and their Anti Bacterial Studies
}

\author{
RAVI KUMAR GANTA ${ }^{1 *}$, MADHU $\mathrm{CH}^{1}$, HYMAVATHI ${ }^{1}$, \\ RAVI KUMAR. M1, M MURALI KRISHNA KUMAR ${ }^{2}$ and VENKATESWARA RAO B ${ }^{1}$
}

${ }^{1}$ Departmaent of Engineering Chemistry, AUCE(A), Andhra University, Visakhapatnam - 530003, India.

${ }^{2} \mathrm{AU}$ Collage of pharmaceutical sciences, Andhra University, Visakhapatnam, 530003, India.

${ }^{*}$ Corresponding author E-mail Id: gantaravichem@gmail.com

http://dx.doi.org/10.13005/ojc/320540

(Received: August 24, 2016; Accepted: October 14, 2016)

\begin{abstract}
A novel and eco friendly procedure for the synthesis of novel 3, 3'-((1, 3-diphenyl-1Hpyrazol-4-yl) methylene) bis (1H-indole) derivatives has been developed through the reaction of 1,3-diphenyl-1H-pyrazole-4-carbaldehyde and $1 \mathrm{H}$-indole using low cost and recyclable Nano copper ferrite catalyst. This novel method offers several advantages, such as high yields, short reaction time, environmental friendly reaction media and recyclable catalyst. The synthesized compounds were confirmed by $\mathrm{H}^{1}, \mathrm{C}^{13} \mathrm{NMR}$ and Mass Spectral analysis. The drug likeness or drugability of all the synthesized compounds were tested through rule of five (RO5) parameters. All the compounds have shown one or more RO5 violations. The compounds were screened for their antibacterial activity against both gram positive and gram negative bacteria. Four of the synthesized compounds ( $3 a, 3 b$, $3 \mathrm{l}$ and $\mathrm{3i}$ ) were found to possess significant antibacterial activity against human pathogenic bacteria. All the four compounds showed RO5 violations.
\end{abstract}

Keywords : Pyrazolyl methylene bis indole, Nano copper ferrite catalyst, Anti bacterial activity, Recyclable catalyst.

\section{INTRODUCTION}

Multicomponent reactions (MCRs) have gained considerable attention due to powerful bond forming capacity in combinatorial and medicinal chemistry ${ }^{1}$. These reactions proceed through onepot procedures to form complex structural target products in a single step. The major advantages of MCRs include shorter reaction time, lower cost, atom-economy, higher conversion, and operational simplicity. MCRs have provided a versatile method for formation of new C-C and C-N bonds in the synthesis of numerous heterocyclic compounds ${ }^{2}$. The nature of the catalyst and solvent also play crucial role in determining yield and selectivity of the product ${ }^{3,4}$. Therefore, development of an inexpensive, mild, and reusable catalyst for MCRs remains a hot topic of interest to the synthetic organic chemist. 
In recent times, considerable attention is paid on pyrazole derivatives due to their interesting biological activities such as analgesic ${ }^{5}$, antipyretic ${ }^{6}$, anti-inflammatory ${ }^{7,8}$, antimicrobial ${ }^{9}$, antiviral ${ }^{10,11}$, antidiabetic ${ }^{12}$, anticancer ${ }^{13,14}$, estrogenic ${ }^{15}$ activity. 1, 3-diphenyl-1H-pyrazole-4-carbaldehyde, one of the starting materials is synthesized in our lab as per the procedure (sceheme1) already laid down ${ }^{16}$. Indole, being a key moiety of many natural products of therapeutic importance, possesses potentially reactive sites for a variety of chemical reactions to generate molecular diversity. Indoles and its derivatives are found in nature and exhibit physiological properties ${ }^{17}$. Bis indolyl metabolites affect the central nerves system and are used as tranquilizers ${ }^{18}$. Various indolyl derivatives display diverse pharmacological activities and are useful in treatment of fibromyalgia, chronic fatigue and irritable bowel syndrome ${ }^{19}$. Vibrindole A (Figure-1), a bisindolylmethane was known to exhibit anti-bacterial activity $^{20}$.

Pyrazolyl methylene bis indoles containing both pyrazole and indole moieties provide novel leading structures for drug-discovery research. However, only a few methodologies have been reported for the synthesis of Pyrazolyl methylene bis indoles ${ }^{21,22}$. Therefore, the development of simple and efficient methods is desirable to generate structurally varied Pyrazolyl methylene bis indoles with a variety of substituents.

One of the best strategies for green and sustainable chemistry is the recovery and reusability of the catalyst, without loss in catalytic activity $^{23}$. Many organic reactions have been carried out by using supported metal catalysis. Magnetic nanocatalyst has great advantage in activity, separation and recyclability ${ }^{23}$. In particular copper ferrite nano particles have been used in many organic transformations such as Ulmann coupling, Sonogashira reaction and C-N, C-S bond formations. Moreover, they also showed good air stability in various organic transformations ${ }^{24}$.

Drug development is expensive involving billions of dollars. A lead should have a range of physico chemical properties that are consistent with the previous record of discovery of orally active compounds. Literature survey reveals that the compounds with poorer physico-chemical properties would fail in the pre-clinical trials. Hence a pass in the physico-chemical properties would preserve both money and time. Before going for antibacterial studies, we have explored drug likeness of the synthesized compounds by following rule of five (RO5). Although the primary aim is to synthesize pyrazolyl methylene bis indole derivatives, we have focused on the physico-chemical properties to test the drug likeness of these compounds so as to set the stage for next phase of development from chemical lead to drug lead.

RO5 has four important parameters that include molecular weight (MW), partition coefficient expressed as logP, number of $\mathrm{H}$-bond donors ( $\mathrm{NH}$ $+\mathrm{OH})$ and number of $\mathrm{H}$-bond acceptors $(\mathrm{N}+\mathrm{O})^{25}$. Lipophilicity is expressed as log of the ratio of octanol solubility to aqueous solubility as described by Moriguchi et $a^{R 6-29}$. An excessive number of $\mathrm{H}$-bond donors may impair permeability of the drug through membranes ${ }^{30,31}$. An excessive number of $\mathrm{H}$-bond acceptors may prevent permeability across a membrane bi-layer. More than $90 \%$ of the oral drugs have a $\mathrm{MW}<500$; $\log \mathrm{P}<5, \mathrm{NH}+\mathrm{OH}<5$ and $\mathrm{N}+\mathrm{O}<10^{24,25}$ Two additional descriptors - total polar surface area (TPSA) and total rotatable bonds (TRB) were also evaluated. TPSA is a powerful descriptor in the characterization of a drug regarding its absorption including gastro-intestinal tract and bioavailability ${ }^{32}$. TRB is another descriptor that gives information about the conformational flexibility of the molecule during the drug's interaction with receptor sites $^{33}$. RO5 parameters combined with TPSA and TRB collectively describe solubility and permeability parameters of a drug.

\section{EXPERIMENTAL}

All experiments were monitored by thin layer chromatography (TLC) performed on precoated silica gel plates. After elution, plate was visualized under UV illumination at $254 \mathrm{~nm}$ for UV active materials. Further visualization was achieved by staining with $\mathrm{KMnO} 4$ and charring on a hot plate. Column chromatography was performed on silica gel (100-200 mesh) by standard techniques. ${ }^{1} \mathrm{HNMR}$ and ${ }^{13} \mathrm{C}$ NMR spectra were recorded on Bruker $300 \mathrm{MHz}$ spectrometer, and $75 \mathrm{MHz}$, using TMS as an internal standard (chemical shifts in $\delta$ ). Peak multiplicities 
of NMR signals were designated as s (singlet), bs (broad singlet), d (doublet), dd (doublet of doublet), t (triplet), $m$ (multiplet) etc. The HRMS spectra were recorded as ESI-HRMS on a Q-TOF LC-MS/MS mass spectrometer. Commercial grade reagents and solvents were used without further purification.

For calculating parameters relating to $\mathrm{RO} 5$ the following approaches were adopted: $\mathrm{m} / \mathrm{z}$ values from mass spectral data was used to arrive at MW; $(\mathrm{NH}+\mathrm{OH})$ and $(\mathrm{N}+\mathrm{O})$ were arrived at by counting<smiles>CC(c1c[nH]c2ccccc12)c1c[nH]c2ccccc12</smiles>

Vibrindole A manually from the structures of the synthesized compounds; logP values along with TPSA and TRB were exclusively taken from molinspiration, an open access package, available on the web. The earlier three parameters were also verified through molinspiration.

Synthesis of 1, 3-diphenyl-1H-pyrazole-4carbaldehyde (Scheme-1) [16]

Substituted phenyl hydrazones were prepared by heating substituted acetophenones with different hydrazines in methanol under reflux for 1-2 h. To a mixture of DMF $(0.1 \mathrm{~mol})$ and phosphorous oxychloride $(0.02 \mathrm{~mol})$, an ice-cold solution of phenyl hydrozone $(0.01 \mathrm{~mol})$ was added drop wise with stirring under cold condition. After the addition, the reaction mixture was refluxed at $60-70^{\circ} \mathrm{C}$ for $4-5 \mathrm{~h}$. Solution was cooled and poured into crushed ice and neutralized with $\mathrm{NaHCO}_{3}$ solution. The solid obtained

Fig. 1: Vibrindole<smiles>[R]c1cc(C(C)=O)cc([R])c1[R]</smiles>

Scheme 1: synthesis of 1, 3-diphenyl-1H-pyrazole-4-carbaldehyde [Ref-16]

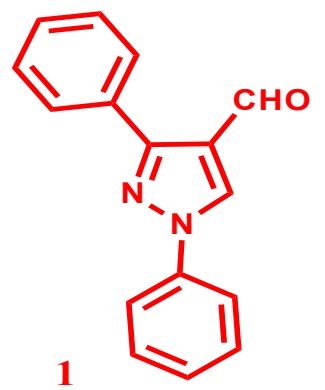<smiles></smiles>

Scheme 2: Optimization for synthesis of 3, 3'-((1, 3-diphenyl-1H-pyrazol-4-yl) methylene) bis (1H-indole) 
was filtered under suction and recrystalized from methanol.

\section{General experimental procedure for synthesis of} pyrazolyl methylene bis indoles

1, 3-diphenyl-1H-pyrazole-4-carbaldehyde (1 $\mathrm{mmol})$, indole (2 mmol), $15 \mathrm{~mol} \%$ CuFeNPS and water as solvent were taken in a $100 \mathrm{ml}$ round bottam flask. The reaction mixture was stirred for $60 \mathrm{~min}$ at $100^{\circ} \mathrm{C}$. The progress of the reaction was monitored by TLC. After the completion of the reaction, the catalyst was separated by using external magnet and reaction mixture was cooled, filterd. The obtained solid product is washed with ethyl acetate before drying over $\mathrm{Na}_{2} \mathrm{SO}_{4}$. After removal of the excess solvent over rotavapour, the desired pyrazolyl methylene bis indoles were obtained in excellent yields. The residual solvents were seperated under vaccum and the products were purified by column chromatography. The identity and purity of the products were confirmed by ${ }^{1} \mathrm{H},{ }^{13} \mathrm{C}$ NMR, and mass spectra.

\section{RESULTS AND DISCUSSION}

\section{Chemistry}

In the present work, we initiated our investigation by screening the reaction of $1 \mathrm{H}$-indole<smiles>[R]c1cc(-c2nn(-c3ccccc3)cc2C=O)cc([R])c1[R]</smiles><smiles>[X]c1ccc2[nH]c(CC(C)C)cc2c1</smiles>

$\mathrm{X}=\mathrm{H}, \mathrm{F}, \mathrm{Cl}, \mathrm{Br}, \mathrm{I}, \mathrm{OMe}$

General Reaction<smiles>[X]c1ccc2[nH]cc(C(c3cn(-c4ccccc4)nc3-c3cc([R])c([R])c([R])c3)c3c[nH]c4ccc([X])cc34)c2c1</smiles>

$3(a-1)$

Scheme 3: synthesis of pyrazolyl methylene bis indole derivatives

Table 1: Optimized Reaction conditions

\begin{tabular}{lccccc}
\hline Entry & solvent & Temperature & Catalyst (mol \%) & Time (min) & Yield \% \\
\hline 1 & - & RT & - & 180 & - \\
2 & Water & RT & - & 120 & - \\
3 & Methanol & RT & - & 120 & - \\
4 & DMSO & RT & - & 120 & - \\
5 & $\mathrm{CH}_{3} \mathrm{CN}$ & $\mathrm{RT}$ & - & 120 & - \\
6 & Water & 60 & 5 & 90 & 11 \\
7 & Methanol & 60 & 5 & 90 & $<10$ \\
8 & DMSO & 60 & 5 & 90 & - \\
9 & $\mathrm{CH}_{3} \mathrm{CN}$ & 60 & 5 & 90 & - \\
10 & Water & 100 & 10 & 60 & 39 \\
11 & Methanol & 100 & 10 & 60 & $<10$ \\
12 & DMSO & 100 & 10 & 60 & $<10$ \\
13 & $\mathrm{CH}_{3} \mathrm{CN}$ & 100 & 10 & 60 & $<10$ \\
14 & Water & 100 & 15 & 60 & 98 \\
15 & Methanol & 100 & 15 & 60 & 16 \\
16 & Water & 130 & 15 & 60 & 98 \\
17 & Water & 100 & 20 & 60 & 98 \\
\hline
\end{tabular}


and 1, 3-diphenyl-1 H-pyrazole-4-carbaldehyde using $\mathrm{CuFe}_{2} \mathrm{O}_{4} \mathrm{NP}$ catalyst in water (Scheme 2). Initially a model reaction was conducted at room temperature without catalyst and solvent. It was observed that no products were obtained even after $3 \mathrm{hr}$ - no new spots observed on TLC plate apart from the reactant spots (Table.1 entry 1). We have investigated the same reaction using different solvents without catalyst at room temperature. It is observed that no products were obtained even after $2 \mathrm{hr}$ (Table. 1 entry 2-5). The same reaction is continued using $5 \mathrm{~mol} \%$ nano $\mathrm{CuFe}_{2} \mathrm{O}_{4} \mathrm{NPs}$ in different solvents, Trace amounts of yields are observed in water and Methanol with same conditions (Table.1 entry 6-7),

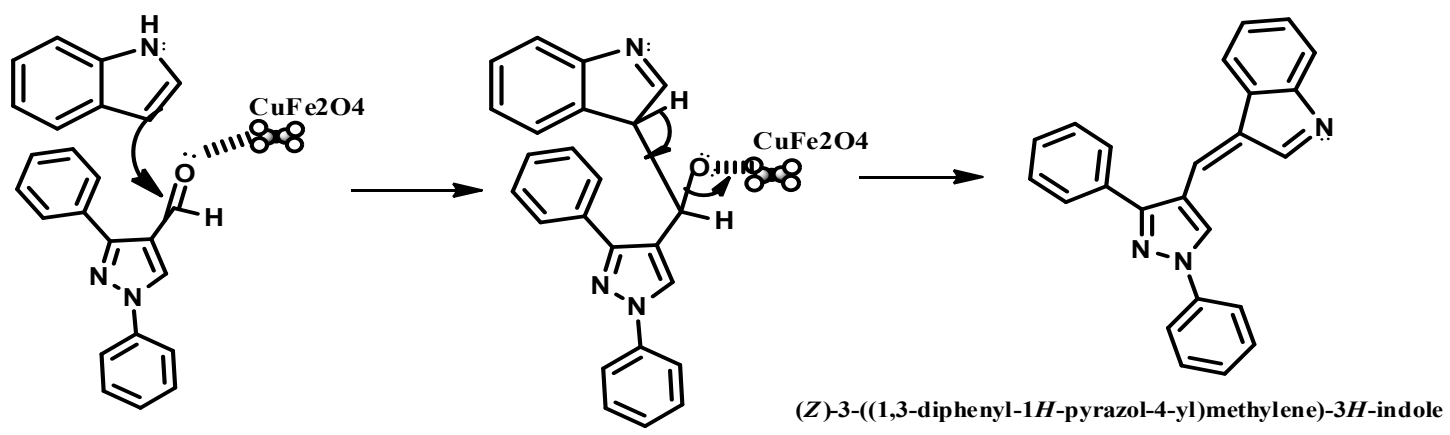

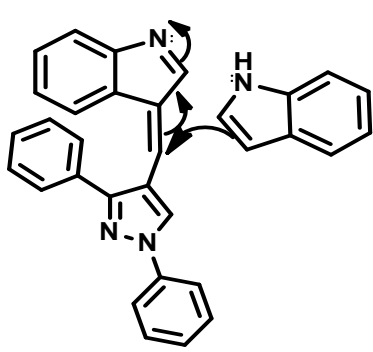

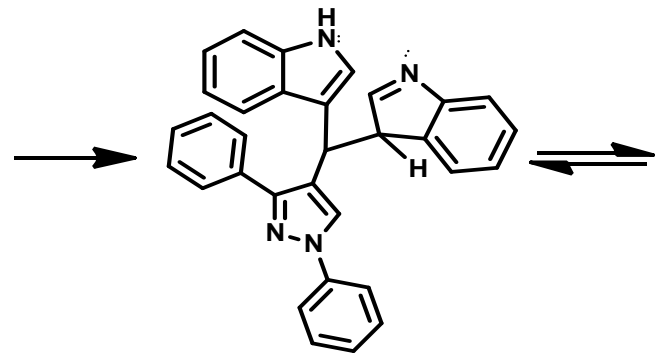<smiles>c1ccc(-c2nn(-c3ccccc3)cc2C(c2c[nH]c3ccccc23)c2c[nH]c3ccccc23)cc1</smiles>

Plausible Mechanism

Fig. 2: Plausible Reaction pathway for synthesis of 3, 3'-((1, 3-diphenyl-1H-pyrazol-4-yl) methylene) bis (1H-indole)

Table 2: synthesis of pyrazolyl methylene bis indole derivatives by using Nano $\mathrm{CuFe}_{2} \mathrm{O}_{4} \mathrm{NPs}$

\begin{tabular}{ccccc}
\hline Code & Pyz aldehydes $(\mathbf{R}=$ & Indoles $(\mathbf{X}=$ & time & yield \\
\hline 3a & $\mathrm{H}$ & $\mathrm{H}$ & 60 & 98 \\
$3 \mathrm{~b}$ & $\mathrm{H}$ & $\mathrm{F}$ & 60 & 94 \\
$3 \mathrm{c}$ & $\mathrm{H}$ & $\mathrm{Cl}$ & 60 & 95 \\
$3 \mathrm{H}$ & $\mathrm{H}$ & $\mathrm{Br}$ & 60 & 93 \\
$3 \mathrm{H}$ & $\mathrm{H}$ & $\mathrm{I}$ & 60 & 89 \\
$3 f$ & $\mathrm{H}$ & $\mathrm{OCH}_{3}$ & 60 & 85 \\
$3 \mathrm{~g}$ & $\mathrm{H}$ & 60 & 85 \\
$3 \mathrm{OCH}$ & $\mathrm{F}$ & 60 & 83 \\
$3 \mathrm{i}$ & $\mathrm{OCH}_{3}$ & $\mathrm{Cl}$ & 60 & 78 \\
$3 \mathrm{j}$ & $\mathrm{OCH}_{3}$ & $\mathrm{Br}$ & 60 & 79 \\
$3 \mathrm{k}$ & $\mathrm{OCH}_{3}$ & $\mathrm{I}$ & 60 & 81 \\
$3 \mathrm{OCCH}$ & $\mathrm{OCH}_{3}$ & $\mathrm{OCH}_{3}$ & 60 & 75 \\
\hline
\end{tabular}


whereas no products were observed in DMSO and $\mathrm{CH}_{3} \mathrm{CN}$ even after $90 \mathrm{~min}$ at $60^{\circ} \mathrm{C}$ (Table. 1 entry 8-9). Greater yield (39\%, Table.1 entry 10 ) was observed with $10 \mathrm{~mol} \% \mathrm{CuFe}_{2} \mathrm{O}_{4} \mathrm{NPs}$ at $100^{\circ} \mathrm{C}$.in water than in other solvents $(<10 \%$, Table. 1 entry $11-13)$ in 60 min. Excellent yields (98\%, Table.1 entry 14) were observed on increasing the catalyst to $15 \mathrm{~mol} \%$ in water solvent for $60 \mathrm{~min}$ at $100{ }^{\circ} \mathrm{C}$. No increase in yield was observed on further increasing the catalyst $\mathrm{mol} \%$.

The model reaction may be summarized as follows: Synthesis of pyrazolyl methylene bis indoles was carried out using 1, 3-diphenyl-1 H-pyrazole4-carbaldehyde, $1 \mathrm{H}$-indole, $15 \mathrm{~mol} \%$ CuFeNPs in water as solvent at $100^{\circ} \mathrm{C}$. Continuing the success, different pyrazolyl aldehydes and different indoles were tested in our attempt to synthesize pyrazolyl methylene bis indoles under the same reaction conditions (Scheme-3) and the results were summarized in table 3.

From the results in table 2, it may be concluded that products in which both pyrazole aldehyde and indole moieties having no substituents (Table 2, 3a-3g) were formed in better yields than methoxy substituted derivatives (Table 2, 3h-3I).). The synthesized compounds were confirmed by $\mathrm{H}^{1}$, $\mathrm{C}^{13}$, NMR and Mass Spectral analysis. The plausible mechanism for the formation of pyrazolyl methylene bis indoles from 1, 3-diphenyl-1 H-pyrazole-4carbaldehyde and $1 \mathrm{H}$-indole using CuFeNPS is shown in figure 2 . The reaction proceeds through the formation of highly reactive, not isolated, (Z)-3-((1, 3-diphenyl-1H-pyrazol-4-yl) methylene)-3H-indole

We have examined the recyclability of the copper ferrite NPs catalyst for the model reaction. The study indicated that catalyst can be reused up to 8 cycles (Table 3 ) under optimized reaction condition without leaching of the $\mathrm{Cu}$ and Fe metals, which is evident from the figure 3. The catalyst was separated by using external magnet after completion of the reaction, washed with pure water followed by ethyl acetate, dried at $100^{\circ} \mathrm{C}$ and reused for the next cycle.

\section{Spectral data}

3a) 3, 3'-((1, 3-diphenyl-1H-pyrazol-4-yl) methylene) bis (1H-indole)

Pale red Solid. M.P: $172-174^{\circ} \mathrm{C}$. ${ }^{1} \mathrm{H}$ NMR (300 MHz, DMSO-d $\left.{ }_{6}\right): \delta 5.39(s, 1 H), 6.84-6.94(m$,

Table 3: Recyclability of catalyst

\begin{tabular}{lllllllll}
\hline Reaction cycle & $\mathbf{1}$ & $\mathbf{2}$ & $\mathbf{3}$ & $\mathbf{4}$ & $\mathbf{5}$ & $\mathbf{6}$ & $\mathbf{8}$ & \\
\hline Yield $(\%)$ & 98 & 96 & 96 & 92 & 91 & 90 & 86 & 81 \\
\hline
\end{tabular}

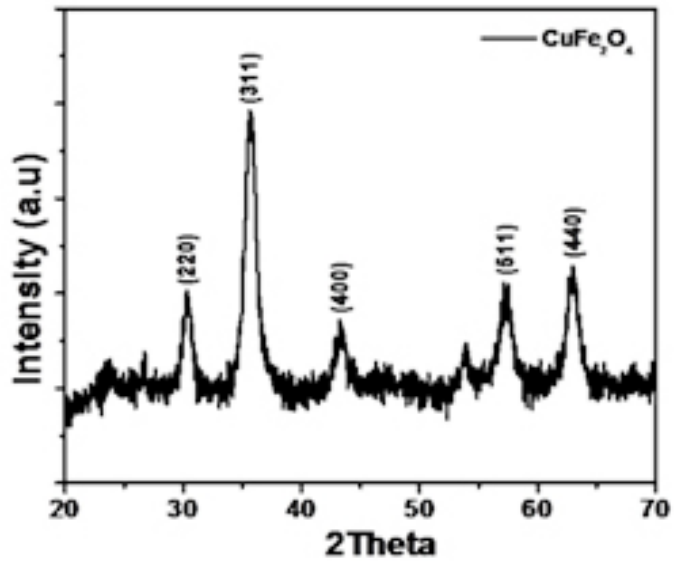

a) Fresh CuFeNPs

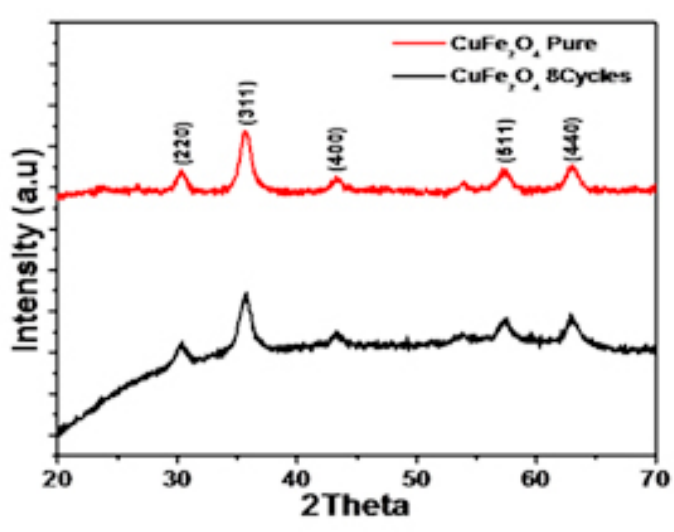

b) After $8^{\text {th }}$ cycle

Fig. 3: XRD pattern of Native CuFeNPs and Reused CuFeNPs 
$4 \mathrm{H})$, 7.01-7.08(m, 2H), 7.18-7.41(m, 9H), 7.667.63(m, 5H), 7.81(s, 1H), 10.39(s, 2H). ${ }^{13} \mathrm{C}$ NMR $(75$ $\left.\mathrm{MHz}, \mathrm{DMSO}-\mathrm{d}_{6}\right): \delta 149.78,139.33,136.53,133.09$, $128.86,127.90,127.27,125.96,125.40,125.18$, 123.23, 120.58, 118.52, 117.94, 117.78, 117.70, 111.13, 29.74. HRMS (ESI) $\mathrm{m} / \mathrm{z}$ : calc. for $\left[\mathrm{M}+\mathrm{H}^{+}\right]$ $\mathrm{C}_{32} \mathrm{H}_{24} \mathrm{~N}_{4}$ : 464.56 ; found: 464.89 . 3b) 3, 3'-((1, 3-diphenyl-1H-pyrazol-4-yl) methylene) bis (5-fluoro-1H-indole

Pale yellow Solid. M.P: $269-271^{\circ} \mathrm{C}{ }^{1} \mathrm{H}$ NMR (300 MHz, DMSO- $\left.\mathrm{d}_{6}\right): \delta 5.84(\mathrm{~s}, 1 \mathrm{H}), 6.87(\mathrm{~d}, \mathrm{~J}=$ $1.88 \mathrm{~Hz}, 2 \mathrm{H}), 7.03(\mathrm{dd}, \mathrm{J}=1.88 \& 8.68 \mathrm{~Hz}, 2 \mathrm{H}), 7.20-$ $7.25(\mathrm{~m}, 3 \mathrm{H}), 7.30-7.43(\mathrm{~m}, 6 \mathrm{H}), 7.61(\mathrm{~d}, \mathrm{~J}=3.021 \mathrm{~Hz}$, $2 \mathrm{H}), 7.65-7.71(\mathrm{~m}, 4 \mathrm{H}), 10.45(\mathrm{~s}, 2 \mathrm{H}) .{ }^{13} \mathrm{C}$ NMR $(75$

Table 4: Solubility and permeability parameters

\begin{tabular}{|c|c|c|c|c|c|c|c|c|}
\hline $\begin{array}{l}\text { Compound } \\
\text { Code }\end{array}$ & $\log P$ & TPSA & $\begin{array}{l}\text { no. of } \\
\text { atoms }\end{array}$ & MW & $\begin{array}{l}\text { No of } \\
\text { H-bond } \\
\text { donors }\end{array}$ & $\begin{array}{c}\text { No of } \\
\text { H-bond } \\
\text { acceptors }\end{array}$ & $\begin{array}{c}\text { No } \\
\text { of } \\
\text { violations }\end{array}$ & $\begin{array}{c}\text { No of } \\
\text { rotatable } \\
\text { bonds }\end{array}$ \\
\hline $3 a$ & 6.72 & 49.41 & 36 & 464.57 & 4 & 2 & 1 & 5 \\
\hline $3 b$ & 5.05 & 49.41 & 38 & 500.55 & 4 & 2 & 2 & 5 \\
\hline $3 c$ & 8.03 & 49.41 & 38 & 533.46 & 4 & 2 & 2 & 5 \\
\hline $3 d$ & 8.26 & 49.41 & 38 & 622.36 & 4 & 2 & 2 & 5 \\
\hline $3 e$ & 8.61 & 49.41 & 38 & 716.36 & 4 & 2 & 2 & 5 \\
\hline $3 f$ & 6.79 & 67.88 & 40 & 524.62 & 6 & 2 & 2 & 7 \\
\hline $3 g$ & 6.35 & 77.11 & 42 & 554.65 & 7 & 2 & 2 & 8 \\
\hline $3 \mathrm{~h}$ & 4.69 & 77.11 & 44 & 590.63 & 7 & 2 & 1 & 8 \\
\hline $3 i$ & 7.66 & 77.11 & 44 & 623.54 & 7 & 2 & 2 & 8 \\
\hline 3j & 7.92 & 77.11 & 44 & 712.44 & 7 & 2 & 2 & 8 \\
\hline $3 k$ & 8.39 & 77.11 & 44 & 806.44 & 7 & 2 & 2 & 8 \\
\hline 31 & 6.42 & 95.58 & 46 & 614.70 & 9 & 2 & 2 & 10 \\
\hline
\end{tabular}

Table 5: antibacterial studies of synthesized compounds

\begin{tabular}{|c|c|c|c|c|c|c|}
\hline \multirow[t]{2}{*}{ Code } & \multirow[t]{2}{*}{ Solubility } & \multirow{2}{*}{$\begin{array}{c}\text { Volume of } \\
\text { sample } \\
\text { tested }\end{array}$} & \multicolumn{2}{|c|}{$\begin{array}{l}\text { Gram +Positive bacteria } \\
\text { (Zone of inhibition in } \mathrm{mm} \text { ) }\end{array}$} & \multicolumn{2}{|c|}{$\begin{array}{l}\text { Gram -Negative bacteria } \\
\text { (Zone of inhibition in } \mathrm{mm} \text { ) }\end{array}$} \\
\hline & & & $\begin{array}{c}\text { Bacillus } \\
\text { Subtilis } \\
\text { (441) }\end{array}$ & $\begin{array}{l}\text { Streptococcus } \\
\text { pyogenes } \\
(442)\end{array}$ & $\begin{array}{c}\text { Klebsiella } \\
\text { pneumonia } \\
\text { (9544) }\end{array}$ & $\begin{array}{c}\text { Escherichia } \\
\text { coli } \\
\text { (E. coli) }\end{array}$ \\
\hline $3 a$ & DMSO & $10 \mu \mathrm{L}$ & 8 & 10 & - & 7 \\
\hline $3 b$ & DMSO & $10 \mu \mathrm{L}$ & 8 & 10 & - & 8 \\
\hline $3 c$ & DMSO & $10 \mu \mathrm{L}$ & 10 & 8 & - & - \\
\hline $3 d$ & DMSO & $10 \mu \mathrm{L}$ & 8 & 8 & - & - \\
\hline $3 e$ & DMSO & $10 \mu \mathrm{L}$ & - & - & - & - \\
\hline $3 f$ & DMSO & $10 \mu \mathrm{L}$ & - & - & - & - \\
\hline $3 g$ & DMSO & $10 \mu \mathrm{L}$ & - & - & - & - \\
\hline $3 \mathrm{~h}$ & DMSO & $10 \mu \mathrm{L}$ & - & - & - & - \\
\hline $3 i$ & DMSO & $10 \mu \mathrm{L}$ & 8 & 8 & - & 6 \\
\hline $3 j$ & DMSO & $10 \mu \mathrm{L}$ & 8 & - & - & - \\
\hline $3 k$ & DMSO & $10 \mu \mathrm{L}$ & - & 9 & - & - \\
\hline 3ા & DMSO & $10 \mu \mathrm{L}$ & 7 & 10 & - & 8 \\
\hline
\end{tabular}


$\left.\mathrm{MHz}, \mathrm{DMSO}-\mathrm{d}_{6}\right): \delta 149.96,139.33,135.03,132.85$ $128.77,127.93,127.23,126.93,125.51,124.84$, $124.33,123.42,120.94,117.93,117.36,112.32$ 29.70. HRMS (ESI) $\mathrm{m} / \mathrm{z}$ : calc. for $\left[\mathrm{M}+\mathrm{H}^{+}\right] \mathrm{C}_{32} \mathrm{H}_{22} \mathrm{~F}_{2} \mathrm{~N}_{4}$ : 500.54; found: 500.98

3c) 3, 3'-((1, 3-diphenyl-1H-pyrazol-4-yl) methylene) bis (5-chloro-1H-indole)

Pale red Solid. M.P: $202-204^{\circ} \mathrm{C}{ }^{1} \mathrm{H}$ NMR $(300 \mathrm{MHz}$, DMSO-d $): \delta 5.84(\mathrm{~s}, 1 \mathrm{H}), 6.82(\mathrm{~d}, \mathrm{~J}=$ $1.88 \mathrm{~Hz}, 2 \mathrm{H}), 7.11-7.32(\mathrm{~m}, 8 \mathrm{H}), 7.36-7.44(\mathrm{~m}$, $5 \mathrm{H}), 7.65-7.71(\mathrm{~m}, 4 \mathrm{H}), 10.12(\mathrm{~s}, 2 \mathrm{H}) .{ }^{13} \mathrm{C}$ NMR $(75$ MHz, DMSO-d $)_{6}$ : $\delta 150.01,139.33,135.25,132.80$, $128.71,127.91,127.62,127.24,125.48,124.61$, $124.22,123.57,121.02,117.98,117.39,112.67$, 111. $27,29.66$.HRMS (ESI) $\mathrm{m} / \mathrm{z}$ : calc. for $\left[\mathrm{M}+\mathrm{H}^{+}\right]$ $\mathrm{C}_{32} \mathrm{H}_{22} \mathrm{Cl}_{2} \mathrm{~N}_{4}: 532.12$; found: 532.74

3d) 3, 3'-((1, 3-diphenyl-1H-pyrazol-4-yl) methylene) bis (5-bromo-1H-indole)

Pale pink Solid. M.P: $256-258^{\circ} \mathrm{C}$. ${ }^{1} \mathrm{H}$ NMR (300 MHz, DMSO-d $)$ : $\delta 5.81(\mathrm{~s}, 1 \mathrm{H}), 6.80-6.90(\mathrm{~m}$, $6 \mathrm{H}), 7.22-7.42(\mathrm{~m}, 7 \mathrm{H}), 7.51(\mathrm{~s}, 1 \mathrm{H}), 7.63-7.72(\mathrm{~m}$, $5 \mathrm{H}), 10.12(\mathrm{~s}, 2 \mathrm{H}) .{ }^{13} \mathrm{C}$ NMR $\left(75 \mathrm{MHz}, \mathrm{DMSO}-\mathrm{d}_{6}\right)$ : $\delta$ 158.04, 154.95, 150.06, 139.36, 133.09, 132.83, 128.66, 127.82, 127.24, 127.12, 126.21, 126.08, $125.38,124.98,124.31,117.91,111.63,111.50$, 109.16, 108.83, 103.56, 103.26, 29.89.HRMS (ESI) $\mathrm{m} / \mathrm{z}$ : calc. for $\left[\mathrm{M}+\mathrm{H}^{+}\right] \mathrm{C}_{32} \mathrm{H}_{22} \mathrm{Br}_{2} \mathrm{~N}_{4}$ : 620.12; found: 620.99

3e) 3, 3'-((1, 3-diphenyl-1H-pyrazol-4-yl) methylene) bis (5-iodo-1H-indole)

White Solid. M.P: $206-208^{\circ} \mathrm{C}{ }^{1} \mathrm{H}$ NMR (300 $\left.\mathrm{MHz}, \mathrm{DMSO}-\mathrm{d}_{6}\right): \delta 5.84(\mathrm{~s}, 1 \mathrm{H}), 6.82(\mathrm{~d}, J=1.88 \mathrm{~Hz}$, $2 \mathrm{H}), 7.17-7.44(\mathrm{~m}, 12 \mathrm{H}), 7.57(\mathrm{~s}, 1 \mathrm{H}), 7.65-7.71(\mathrm{~m}$, $4 \mathrm{H}), 10.08(\mathrm{~s}, 2 \mathrm{H}) \cdot{ }^{13} \mathrm{C}$ NMR $\left(75 \mathrm{MHz}\right.$, DMSO- $\left.\mathrm{d}_{6}\right)$ : $\delta$ 158.87, 155.78, 150.89, 140.18, 133.92, 133.66, $129.49,128.66,128.07,127.95,127.04,126.91$, $126.21,125.81,125.14,118.74,112.46,112.33$, $109.99,109.66,104.49,104.09$, 30.72.HRMS (ESI) $\mathrm{m} / \mathrm{z}$ : calc. for $\left[\mathrm{M}+\mathrm{H}^{+}\right] \mathrm{C}_{32} \mathrm{H}_{22} \mathrm{I}_{2} \mathrm{~N}_{4}: 716.64$; found: 716.99

3f) 3, 3'-((1,3-diphenyl-1H-pyrazol-4-yl) methylene) bis (5-methoxy-1H-indole)

Pale pink Solid. M.P: $198-200^{\circ} \mathrm{C}{ }^{1} \mathrm{H}$ NMR $\left(300 \mathrm{MHz}, \mathrm{DMSO}-\mathrm{d}_{6}\right): \delta 3.55(\mathrm{~s}, 6 \mathrm{H}), 5.80(\mathrm{~s}, 1 \mathrm{H})$, 6.65-6.71 (m, 4H), 6.99 (d, J=2.07 Hz, 2H), 7.22-7.28 (m, 3H), 7.33- 7.47(m, 5H), 7.68(d, J=6.42 Hz, 2H), 7.84(d, J=7.74 Hz, 2H), 8.19(s,1H), 10.69(s, 2H). ${ }^{13} \mathrm{C}$ NMR (75 MHz, DMSO-d d : $\delta 152.61,150.04,139.44$, $133.25,131.70,129.33,128.36,127.85,127.68$, $126.50,125.80,125.50,124.19,117.91,117.55$, 112.04, 110.50, 100.66, 55.06, 30.56, 29.50. HRMS (ESI) $\mathrm{m} / \mathrm{z}$ : calc. for $\left[\mathrm{M}+\mathrm{H}^{+}\right] \mathrm{C}_{34} \mathrm{H}_{24} \mathrm{~N}_{4} \mathrm{O}_{2}: 524.22$; found: 524.91.

3g) 3, 3'-((1-phenyl, 3-3, 4, 5 tri methoxy phenyl1H-pyrazol-4-yl) methylene) bis (1H-indole) White Solid. M.P: $233-236^{\circ} \mathrm{C}{ }^{1} \mathrm{H}$ NMR (300 $\left.\mathrm{MHz}, \mathrm{DMSO}-\mathrm{d}_{6}\right): \delta 2.57-2.96(\mathrm{~m}, 6 \mathrm{H}), 3.72(\mathrm{~s}, 3 \mathrm{H})$, 5.92(s, 1H), 6.89-6.93(m, 6H), 7.06(t, J= 7.36 \& $15.10 \mathrm{~Hz}, 2 \mathrm{H}), 7.21(\mathrm{t}, \mathrm{J}=7.17 \& 14.54 \mathrm{~Hz}, 1 \mathrm{H}), 7.32-$ 7.42(m, 6H), 7.66-7.91 (m,3H), 10.52 (brs, $2 \mathrm{H}) .{ }^{13} \mathrm{C}$ NMR (75 MHz, DMSO-d $)$ : $\delta 152.59,149.51,139.39$, 137.01, 136.70, 129.15, 128.58, 127.73, 126.08, 125.62 , 125.00, 123.65, 120.86, 118.60, 118.15, 117.88, 111.36, 104.56, 59.83, 54.92, 30.06. HRMS (ESI) $\mathrm{m} / \mathrm{z}$ : calc. for $\left[\mathrm{M}+\mathrm{H}^{+}\right] \mathrm{C}_{35} \mathrm{H}_{30} \mathrm{~N}_{4} \mathrm{O}_{3}: 555.64$; found: 555.09

3h) 3, 3'-((1-phenyl, 3-3, 4, 5 tri methoxy phenyl$1 \mathrm{H}-$ pyrazol-4-yl) methylene) bis (5-fluoroindole)

Pale pink Solid. M.P: $284-286^{\circ} \mathrm{C}{ }^{1} \mathrm{H}$ NMR $\left(300 \mathrm{MHz}, \mathrm{DMSO}-\mathrm{d}_{6}\right): \delta 3.41(\mathrm{~s}, 6 \mathrm{H}), 3.79(\mathrm{~s}, 3 \mathrm{H})$, $5.84(\mathrm{~s}, 1 \mathrm{H}), 6.87-6.92(\mathrm{~m}, 4 \mathrm{H}), 7.06(\mathrm{dd}, J=2.07$ \& $8.68 \mathrm{~Hz}, 2 \mathrm{H}), 7.20-7.44(\mathrm{~m}, 7 \mathrm{H}), 7.54(\mathrm{~s}, 1 \mathrm{H}), 7.66(\mathrm{~d}$, $\mathrm{J}=7.74 \mathrm{~Hz}, 2 \mathrm{H}), 10.14(\mathrm{~s}, 2 \mathrm{H}) \cdot{ }^{13} \mathrm{C}$ NMR $(75 \mathrm{MHz}$, DMSO-d $\left.\mathrm{d}_{6}\right): \delta 152.42,149.58,139.25,136.87$, $135.03,128.75,128.30,127.28,126.85,125.48$, $125.05,124.07,123.49,121.02,117.90,117.68$, 117.33, 112.34, 104.24, 60.01, 56.63, 54.81, 29.82. HRMS (ESI) $\mathrm{m} / \mathrm{z}$ : calc. for $\left[\mathrm{M}+\mathrm{H}^{+}\right] \mathrm{C}_{35} \mathrm{H}_{28} \mathrm{~F}_{2} \mathrm{~N}_{4} \mathrm{O}_{3}$ : 591.92; found: 591.06

3i) 3, 3'-((1-phenyl, 3-3, 4, 5 tri methoxy phenyl-1Hpyrazol-4-yl) methylene) bis (5-chloro-indole) White Solid. M.P: $212-215^{\circ} \mathrm{C}{ }^{1} \mathrm{H}$ NMR (300 MHz, DMSO-d $)$ : $\delta 3.41(\mathrm{~s}, 6 \mathrm{H}), 3.79(\mathrm{~s}, 3 \mathrm{H})$, 5.83(s, 1H), 6.86-6.91(m, 4H), 7.16-7.25(m, 3H), $7.30(\mathrm{~d}, \mathrm{~J}=8.8 \mathrm{~Hz}, 2 \mathrm{H}), 7.37-7.46(\mathrm{~m}, 4 \mathrm{H}), 7.54(\mathrm{~s}$, $1 \mathrm{H}), 7.67(\mathrm{~d}, \mathrm{~J}=7.74 \mathrm{~Hz}, 2 \mathrm{H}), 10.43(\mathrm{~s}, 2 \mathrm{H}) .{ }^{13} \mathrm{C}$ NMR $\left(75 \mathrm{MHz}, \mathrm{DMSO}-\mathrm{d}_{6}\right): \delta 152.46,149.50,136.94$, $135.33,128.85,128.33,127.58,127.31,125.54$, $125.00,124.16,123.52,120.67,117.89,117.21$, 113.01, 111.13, 104.32, 59.95, 54.86, 29.76. HRMS (ESI) $\mathrm{m} / \mathrm{z}$ : calc. for $\left[\mathrm{M}+\mathrm{H}^{+}\right] \mathrm{C}_{35} \mathrm{H}_{28} \mathrm{Cl}_{2} \mathrm{~N}_{4} \mathrm{O}_{3}$ : 623.53; found: 623.03 
3j) 3,3'-((1-phenyl, 3-3, 4, 5 tri methoxy phenyl-1Hpyrazol-4-yl) methylene) bis (5-bromo-indole)

Pale pink Solid. M.P: $158-160^{\circ} \mathrm{C} .{ }^{1} \mathrm{H}$ NMR $\left(300 \mathrm{MHz}, \mathrm{DMSO}-\mathrm{d}_{6}\right): \delta 2.98(\mathrm{~s}, 6 \mathrm{H}), 3.78(\mathrm{~s}, 3 \mathrm{H})$, 5.80(s, $1 \mathrm{H}), 6.81-6.97(\mathrm{~m}, 8 \mathrm{H}), 7.22(\mathrm{t}, \mathrm{J}=7.36$ \& $14.73 \mathrm{~Hz}, 1 \mathrm{H}), 7.29-7.42(\mathrm{~m}, 4 \mathrm{H}), 7.57(\mathrm{~s}, 1 \mathrm{H})$, $7.66(\mathrm{~d}, \mathrm{~J}=7.74 \mathrm{~Hz}, 2 \mathrm{H}), 10.17(\mathrm{~s}, 2 \mathrm{H}) \cdot{ }^{13} \mathrm{C}$ NMR $\left(75 \mathrm{MHz}, \mathrm{DMSO}-\mathrm{d}_{6}\right): \delta 158.08,154.99,152.41$, $149.72,139.30,136.83,133.17,128.71,128.34$, $127.35,126.16,126.02,125.44,125.28,124.06$, $117.91,117.71,111.79,111.67,109.28,108.94$, 104.22, 103.41, 103.10, 60.05, 54.78, 30.12. HRMS (ESI) $\mathrm{m} / \mathrm{z}$ : calc. for $\left[\mathrm{M}+\mathrm{H}^{+}\right] \mathrm{C}_{35} \mathrm{H}_{28} \mathrm{Br}_{2} \mathrm{~N}_{4} \mathrm{O}_{3}: 712.43$; found: 712.82

3k) 3, 3'-((1-phenyl, 3-3, 4, 5 tri methoxy phenyl1H-pyrazol-4-yl) methylene) bis (5-iodo-indole)

Light yellow solid. M.P: $172-174^{\circ} \mathrm{C} .{ }^{1} \mathrm{H}$ NMR $\left(300 \mathrm{MHz}, \mathrm{DMSO}-\mathrm{d}_{6}\right): \delta 3.41(\mathrm{~s}, 6 \mathrm{H}), 3.79(\mathrm{~s}, 3 \mathrm{H})$, 6.87-6.92(m, $4 \mathrm{H}), 7.04-7.08(\mathrm{~m}, 2 \mathrm{H}), 7.20-7.43(\mathrm{~m}$, $8 \mathrm{H}), 7.54(\mathrm{~s}, 1 \mathrm{H}), 7.66(\mathrm{~d}, \mathrm{~J}=8.8 \mathrm{~Hz}, 2 \mathrm{H}), 10.14(\mathrm{~s}, 2 \mathrm{H})$. ${ }^{13} \mathrm{C}$ NMR $(75 \mathrm{MHz}$, DMSO-d $): \delta 153.2,150.4$, $140.1,137.7,135.9,129.6,129.1,128.1,127.7$, $126.3,125.9,124.9,124.3,121.8,118.7,118.5$, 118.2, 113.2, 105.1, 60.8, 57.5, 55.6, 30.6. HRMS (ESI) $\mathrm{m} / \mathrm{z}$ : calc. for $\left[\mathrm{M}+\mathrm{H}^{+}\right] \mathrm{C}_{35} \mathrm{H}_{28} \mathrm{l}_{2} \mathrm{~N}_{4} \mathrm{O}_{3}: 807.03$; found: 807.14

3I) 3, 3'-((1-phenyl, 3-3, 4, 5 tri methoxy phenyl-1Hpyrazol-4-yl) methylene) bis (5-methoxy-indole) White Solid. M.P: $202-206^{\circ} \mathrm{C}{ }^{1} \mathrm{H}$ NMR (300 MHz, DMSO-d $\left.\mathrm{d}_{6}\right): \delta 3.38(\mathrm{~s}, 6 \mathrm{H}), 3.66(\mathrm{~s}, 6 \mathrm{H})$, 3.77(s, 3H), 5.82(s, 1H), 6.74-6.84(m, 6H), 6.97(s, $2 \mathrm{H}), 7.19-7.29(\mathrm{~s}, 3 \mathrm{H}), 7.39(\mathrm{t}, \mathrm{J}=8.12 \& 15.67 \mathrm{~Hz}$, $2 \mathrm{H}), 7.57-7.67(\mathrm{~m}, 3 \mathrm{H}), 9.97(\mathrm{~s}, 2 \mathrm{H}) .{ }^{13} \mathrm{C}$ NMR $(75$ $\mathrm{MHz}$, DMSO- $\left.\mathrm{d}_{6}\right): \delta 152.79,152.46,149.85,139.44$, $136.85,131.96,128.78,128.63,127.56,126.42$, $125.41,124.68,124.30,117.96,117.69,111.66$, 110.67, 104.38, 101.14, 60.11, 55.37, 54.89, 30.16.

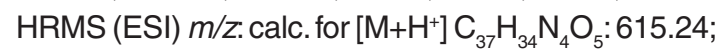
found: 615.07

\section{Drug likeness through RO5}

The drug likeness or drugability of all the synthesized compounds were tested through RO5 parameters. All the compounds have shown one or two RO5 violation (Table 4). These values were correlated with obtained antibacterial activities. It was found that compounds showing RO5 violations have very poor or no antibacterial activity. The descriptors TPSA, TRB were included for the sake of future interpretations and were not detailed here.

\section{Biology (antibacterial activity)}

Antibacterial activity assay was performed by Disk-diffusion method. Antibacterial activities of synthesized compounds were screened against two gram positive bacteria (Bacillus subtilis, Streptococcus pyogenes) and two gram negative bacteria (klebsiella pneumonia, Escherichia coll). The anti bacterial activity was determined by measuring zone of inhibition in millimeter and compared with standard drug, streptomycin. Compounds $3 \mathrm{a}, 3 \mathrm{~b}, 3 \mathrm{i}$, and 31 showed moderate antibacterial activity against gram + Ve (Bacillus subtilis and Streptococcus pyogenes) and gram negative bacteria (E. coll). The results were summarized in table 5

\section{CONCLUSION}

In summary, a new method has been developed using CuFeNPs as catalyst in aqueous media at $100^{\circ} \mathrm{C}$ for the synthesis of novel pyrazolyl methylene bis indoles. This procedure showed good functional group tolerance. Other features of this protocol are, short reaction time, operational simplicity, higher yields, avoiding the use of organic solvents. The catalyst is easily separated by using external magnet and it is reusable up to eight cycles. The drug likeness or drugability of all the synthesized compounds were tested through rule of five (RO5) parameters. All the compounds have shown one or more RO5 violations. Furthermore, the compounds are screened for their antibacterial activity against human pathogenic bacteria. Compounds $3 a, 3 b, 3 i$, and 3 l showed good antibacterial activity against human pathogenic bacteria

\section{ACKNOWLEDGMENTS}

The Corresponding author is grateful to CSIR, New Delhi for supporting through fellowship (JRF\&SRF), and Department of Engineering chemistry, AUCE (A), Andhra University, Visakhapatnam for providing general lab facilities. The author is also thankful to Prof. B. Venkateswara Rao for his valuable and constant support. 


\section{REFERENCES}

1. (a) Zhu, J.; Bienayme, H. Multicomponent Reactions, 1st ed.; Wiley-VCH: Weinheim, Germany, 2005; (b) Dömling, A. Chem. Rev. 2006, 106, 17; (c) Zhu, J. Eur. J. Org. Chem. 2003, 1133; (d) Dömling, A.; Ugi, I. Angew. Chem., Int. Ed. 2000, 39, 3168.

2. (a) Ghosh, A. K.; Kulkarni, S.; Xu, C.; Fanwick, P. E. Org. Lett. 2006, 8, 4508 (b) Fedou, N. M.; Parsons, P. J.; Viseux, E.; Whittle, A. J. Org. Lett. 2005, 7, 3179 (c) Ma, C.; Yang, Y.W. Org. Lett. 2005, 7, 1343; (d) Pirrung, M. C.;Sarma, K. D. J. Am. Chem. Soc. 2004, 126, 444; (e) Neumann, H. J. Am. Chem.Soc. 2001, 123 , 8398; (f) Byk, G.; Gottlieb, H. E.; Herscovici, J.; Mirkin, F. J.Comb. Chem. 2000, 2, 732.

3. Wang, X.-S.; Li, Q.; Wu, J.-R.; Li, Y.-L.; Yao, C.-S.; Tu, S.-J. Synthesis. 2008, 1902.

4. Khan, A. T.; Khan, M. M.; Bannuru, K. K. R. Tetrahedron. 2010, 66, 7762;

5. Sahu, S. K.; Banerjee, M.; Azam, M. A. Trop J Pharm Res., 2008, 7(2), 961.

6. Fabiane, R. S.; Vanessa, T. S.; Lysandro, P. B.; Viviane, R.; Marli, R. O.; Helio, G. B.; Nilo, Z.; Marcos, A. P. M.; Carlos, F. M. Eur J Pharmacol., 2002, 451(2), 141.

7. Makhsumov, A. D.; Kilichov, G.; Nikbaev, A. T. Pharm Chem J. 1986, 20, 289.

8. Bekhit, A. A.; Ashour, H. M.; Guemei, A. A. Arch Pharm(Weinheim), 2005, 338(4), 167.

9. Shak, B.; Ahmet, S.; Ekrem, A.; Abdulhamit, B. Med Chem Res. 2009, 18(5), 327.

10. Brahmbhatt, D. I.; Ankit, R. K.; Anil, K. P.; Niraj, H. P. Indian J Chem. 2010, 49B,971.

11. Guiping, O.; Xue-Jian, C.; Zhuo, C.; Bao-An, S.; Pinaki, S. B.; Song, Y.; Lin-Hong, J.; Wei, X.; De-Yu, H.; Song, Z. J Agric Food Chem. 2008,56(21), 10160.

12. Prakash, O.; Rashmi, P.; Pooja, R.; Kamaljeet, P.; Yogita, D.; Aneja, K. R. Indian J Chem. 2009, 48B, 563.

13. Bandgar, B. P.; Totre, J. V.; Gawande, S. S.; Khobragade, C. N.; Warangkar, S. C.; Kadam, P. D. Bioorg Med Chem. 2010, 18(16), 6149.

14. Nusrat, B. A.; Rabiul, M. I. Bangladesh J Pharmacol., 2007, 2, 81.

15. Fotini, N.; Konstantinos, M. K.; Prokopios, M.; Serkos, A. Molecules. 2007, 12(7), 1259.

16. Deepa, M.; Harinadha babu, V.; Parameshwar,
R.; Madhava Reddy, B. E-Journal of Chemistry, 2012, 9(1), 420.

17. (a) Sundberg, R. J. Indoles; Academic Press: San Diego, 1996; (b) Faulkner, D. J. Nat. Prod. Rep. 2001, 18, 1; (c) Ninomiya, I. J. Nat. Prod. 1992, 55, 541

18. (a) Foldeak, S.; Czombas, J.; Matkovis, B. Acta Univ.Sjeged. Acta Phys. Chem. 1965, 11, 115; (b) Povszasz, J.;Katalin, G. P.; Foleat, S.; Malkovics, B. Acta Phys. Acad.Sci. Hung. 1996, 29, 299.

19. (a) Kathleen, A.; Merrill, A.G. PCT Int Appl. WO 99,Chem. Abstr. 1999, 130, 276765; (b) Bradfield, C. A.; Bjeldanes, L. F. J. Toxicol. Environ. Health. 1987, 21,311; (c) Dashwood, R. H.; Uyetake, L.; Fong, A. T.;Hendricks, J. D.; Bailey, G. S. Food Chem. Toxicol.1987, 27, 385.

20. Bell, R.; Shmjel, C. Jouml of Natural Pmdurts. 1994, 57, 1587.

21. Sivaprasad, G.; Perumal, P. T.; Prabavathy, V. R.; Mathivananb, N. Bioorganic \& Medicinal Chemistry Letters. 2006, 16, 6302.

22. Farhanullah.; Sharon, A.; Maulik, P. R.; Ram, V. J. Tetrahedron Letters .2004, 45, 5099

23. Baig, R. B. N.; Varma, R. S. Chem. Commun. 2013, 752.

24. (a) Dandia, A.; Jain, A. K.; Sharma, S. RSC Adv. 2013, 3, 2924; (b) Panda, N.; Jena, A. K.; Mohapatra, S.; Rout, S. R. Tetrahedron Lett. 2011, 52, 1924 (c)Bazgir, A.; Hosseini, G.; Ghahremanzadeh, R. ACS Comb. Sci. 2013, 15, 530;(d) Tasca, J. E.; Ponzinibbio, A.; Diaz, G.; Bravo, R. D.; Lavat, A.; Gonza' lez, M. G. Top. Catal. 2010, 53, 1087.

25. Lipinski, C. A. Drug Discovery Today: Technologies, 2004, 1.4, 337.

26. Bernard, T.; Pierre-Alain, C.; Patrick, G.; Frédéric, B.; Peter, W. Pharm research. 1996, 13. 3, 335

27. Moriguchi, I.; Hirono, S.; Liu, Q.; Nakagome, Y; Matsushita, Y. Chem. Pharm. Bull. 1992, 40, 127.

28. Moriguchi, I.; Hirono, S.; Nakagome, I; Hiram, H. Chem. Pharm. Bull. 1994, 42, 976.

29. Leo, A.J. Chem. Pharm. Bull. 1995, 43, 512.

30. Abraham, M.H.; Chadha, S. H.; Whiting, G.S.; Mitchell, R.C. J. Pharm. Sci. 1994, 83, 
1085.

31. Paterson. D.A.; Conradi, R.A.; Hilgers, A.R.; Vidmar, T.J.; Burton, P.S. Quant. Struct:Act. Relatsh. 1994, 13, 4.
32. Ertl, P.; Rohde, B.; Selzer, P. J.Med.Chem. 2000, 43, 3714.

33. Veber, D.F.; Johnson, S. R.; Cheng, H.-Y.; Smith, B.R.; Ward, K.W.; Kopple, K.D. J.Med. Chem. 2002, 45, 2615. 